\title{
A fast autofocus algorithm for synthetic aperture radar processing
}

\author{
Dall, Jørgen
}

Published in:

I E E E International Conference on Acoustics, Speech and Signal Processing. Proceedings

Link to article, DOI:

10.1109/ICASSP.1992.226290

Publication date:

1992

Document Version

Publisher's PDF, also known as Version of record

Link back to DTU Orbit

Citation (APA):

Dall, J. (1992). A fast autofocus algorithm for synthetic aperture radar processing. I E E E International Conference on Acoustics, Speech and Signal Processing. Proceedings, 3, 5 - 8.

https://doi.org/10.1109/ICASSP.1992.226290

\section{General rights}

Copyright and moral rights for the publications made accessible in the public portal are retained by the authors and/or other copyright owners and it is a condition of accessing publications that users recognise and abide by the legal requirements associated with these rights.

- Users may download and print one copy of any publication from the public portal for the purpose of private study or research.

- You may not further distribute the material or use it for any profit-making activity or commercial gain

- You may freely distribute the URL identifying the publication in the public portal

If you believe that this document breaches copyright please contact us providing details, and we will remove access to the work immediately and investigate your claim 


\title{
A FAST AUTOFOCUS ALGORITH FOR \\ SYNTHETIC APERTURE RADAR PROCESSING
}

\author{
Jørgen Dall
}

Electromagnetics Institute,

Technical University of Denmark,

DK-2800 Lyngby, Denmark

\section{ABSTRACT}

Synthetic Aperture Radar (SAR) has become a valuable instrument with applications in reconnaissance and remote sensing. High resolution SAR imaging requires the motion of the radar platform to be known very accurately. Otherwise phase errors are induced in the processing of the raw SAR data, and bad focusing results. In particular, a constant error in the measured along track velocity or the cross track acceleration leads to a phase error that varies quadraticly over the synthetic aperture. The process of estimating this quadratic phase error directly from the radar data is termed autofocus. This paper presents a novel autofocus algorithm with a computational complexity which is at least an order of magnitude lower than that of other algorithms providing comparable accuracies. The new algorithm has been tested on data from the Danish airborne SAR, and the performance has been compared with that of the traditional map drift algorithm.

\section{INTRODUCTION}

The two traditional autofocus algorithms call for comprehensive computations because they require the image to be processed repeatedly. Consequently, these algorithms are not very suitable for real-time processing, and they are usually applied to only a sub-window of the scene. The contrast optimization algorithm [1 is in principle a trial and error method. Data are processed using a number of different velocities, and the velocity leading to the highest contrast is taken as the optimum. The map drift algorithm [2] takes advantage of the fact that two looks do not register properly unless the correct velocity is used for the processing. The misregistration is measured by cross-correlating the processed looks and finding the position of the cross-correlation peak. However, in order to translate the peak position into a velocity error the procedure must be repeated for a number of different processing velocities.

The autofocus algorithm presented in this paper[3] is called the shift-and-correlate (SAC) algorithm since its basic operations are a frequency shift and a cross-correlation. The principle is illustrated in Fig. 1. The Doppler history associated with a point target is a linearly frequency modulated signal. The derivative of the instantaneous frequency, the Doppler rate, is proportional to the square of the along track velocity (for zero cross track acceleration), and so an estimate of this velocity can be obtained from an estimate of the Doppler rate. The SAC algorithm forms two looks by band pass filtering the Doppler signal. One look is frequency shifted and cross-correlated by the other look. The cross-correlation leads to a pulse compression (the time reverse and complex conjugate of one look serves as a matched fitter to the other), and the desired Doppler rate is obtained from the position of the resulting peak and the frequency shift applied.

It is emphasized that in the SAC algorithm the looks are not focused and detected prior to the cross-correlation, so although a

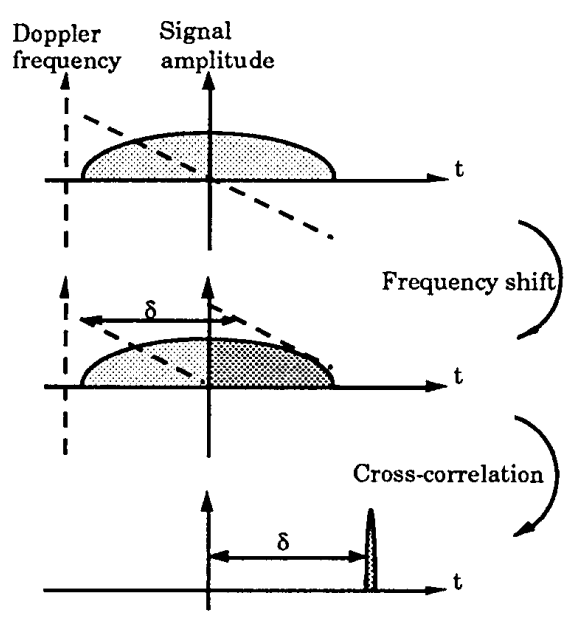

Fig. 1. SAC principle.

look correlation is also involved in the map drift algorithm, the two algorithms are fundamentally different. No image formation takes place at any point of the SAC algorithm, even not as a result of the above-mentioned pulse compression. This is due to the fact that the position of the cross-correlation peak is independent of the target azimuth position, meaning that the cross-correlation functions associated with the individual targets at the same range coincide.

A speckle-like noise is imposed to the resulting cross-correlation function because its contributions from different point targets do not add up coherently. Furthermore look 1 from one target also correlates with look 2 from other targets and gives contributions which do not coincide. Still, the resulting correlation function does have a maximum at time $t=\Delta$, if the scene is inhomogeneous, i.e. has an autocorrelation function with a well-defined peak at $t=0$. This is demonstrated in Section 3 .

Section 4 outlines the implementation of the SAC algorithm, and Section 5 discusses its performance. Finally, a few conclusions are made.

\section{SAC THEORY}

The SAC algorithm and the map drift algorithm have similar performances in the sense that the ensemble average of the detected $\mathrm{SAC}$ correlation function is almost the same as the correlation function resulting from the map drift algorithm. To show this a general scene model is defined.

\section{III-5}


For a scene consisting of a set of point scatterers with complex radar cross sections having independent and uniformly distributed phases from 0 to $2 \pi$, it can be justified [4], that the complex backscatter coefficient per unit area $\sigma_{c}(r)=\sigma_{c}(x, y)$ is a white-noise complex circular symmetric Gaussian process with the autocorrelation function

$$
R_{\sigma_{c}}\left(r_{0}, r_{0}+r\right)=E\left\{\sigma^{\circ}\left(r_{0}\right) \sigma^{\circ}\left(r_{o}+r\right\}\right\}=\delta(r) \sigma^{\circ}\left(r_{0}\right)
$$

Here $\delta()$ is the two-dimensional Dirac delta function, $\sigma^{\circ}=$ $E\left\{\left(\left.\sigma_{c}\right|^{2}\right\}\right.$ is the real backscatter coefficient, and $E\{\}$ denotes ensemble average. It is assumed that many scatterers are present within the radar impulse response.

In the following only one-dimensional correlations in the azimuth direction are of interest, e.g. the autocorrelation function for the scene backscatter coefficient

$R_{\sigma^{\prime}}(t)=E\left\{\int \sigma^{\circ}\left(t_{0}\right) \sigma^{\circ}\left(t_{o}+t\right) d t_{o}\right\}$

Here the along track speed, $v$, of the SAR platform relates the time parameter $\mathrm{t}=\mathrm{vx}$ to the spatial azimuth parameter, $\mathrm{x}$.

The Doppler signal, $s$, associated with a scatterer at $t_{0}$ is assumed to be linearly frequency modulated, i.e. higher order motion errors are neglected or eliminated by correction, so that only a quadratic phase error remains

$$
\begin{aligned}
s\left(t, t_{0}\right)= & \sigma_{c}\left(t_{o}\right) a\left(t-t_{o}-\frac{f_{D C}}{f_{D R}}\right) . \\
& \exp \left(j 2 \pi \frac{1}{2} f_{D R}\left(t-t_{o}\right)^{2}\right)
\end{aligned}
$$

$f_{D R}$ and $f_{D C}$ are the Doppler rate and the Doppler centroid, respectively, and $a_{t}$ represents the (real) weighting imposed by the antenna (and a potential azimuth pre-summer). For small angles the antenna azimuth pattern, $a_{\phi}(\phi)$, can be mapped to the time domain by $a_{t}(t)=a_{\phi}\left(k_{1} \phi\right)$, and likewise for large time bandwidth products the principle of stationary phase allows a mapping to the frequency domain. $a_{f}(\phi)=a_{\phi}\left(k_{2} \phi\right)$.

Now, two signals are generated by band-pass filtering. These signals correspond to the spectrum below and above the Doppler centroid, and they are frequency shifted by $+f_{o}$ and $-f_{o}$, respectively. The signals to be correlated are $s_{-}(t)$ and $s_{+}(t)$, where

$$
\begin{aligned}
s .(t)= & \int \sigma_{c}\left(b_{0}\right) a_{t}-\left(t-t_{o}-\frac{f_{D C}}{f_{D R}}\right) . \\
& \exp \left(j 2 \pi\left(\frac{1}{2} f_{D R}\left(t-t_{o}\right)^{2}+f_{o} t\right)\right) d t_{o}
\end{aligned}
$$

and $s_{+}(t)$ is similar except for a substitution of $a_{t+}$ and $-f_{o}$ for $a_{t-}$ and $+f_{o}$, respectively. $a_{t-}(t)=a_{t}(t)$ for $t<0$ and otherwise 0 , while $a_{t+}(t)=a_{t}(t)$ for $t \geq 0$ and otherwise 0 .

With

$f_{o}=\frac{1}{2} f_{D R} \Delta$

the result of the cross-correlation becomes

$$
\begin{aligned}
& s_{c}(\tau)=\iint \sigma_{c}^{*}\left(t_{0}\right) \sigma_{c}\left(t_{1}\right) s_{p}\left(\tau-\Delta+t_{0}-t_{1}\right) . \\
& \left.\exp \left(j 2 \pi d f_{D C}\left(\tau-\Delta+t_{0}-t_{1}\right)-\frac{1}{2} f_{D R} \Delta\left(t_{0}+t_{1}\right)\right)\right) d t_{0} d t_{1}
\end{aligned}
$$

where

$s_{p}(v)=\int a_{q}+\left(u+\frac{v+\Delta}{2}\right) a_{t}\left(u-\frac{v+\Delta}{2}\right)$.

$\exp \left(\hat{\left.2 \pi f_{D R} u v\right) d u}\right.$

is the compressed pulse corresponding to Fig. 1. By using the principle of stationary phase, it is seen that $s_{p}(v)$ peaks at $v=0$, and that the peak width is inversely proportional to the effective width, $B_{e}$, of $a_{i+}(u+\Delta / 2) a_{i-}(u-\Delta / 2)$. This means that the accuracy of the Doppler rate estimate improves proportionally to $\Delta \mathrm{B}_{e}$.

By using Eq. 1 the ensemble average of $s_{c}(\tau)$ is found to be

$$
\begin{aligned}
E\left\{s_{c}(\tau)\right\} & =s_{p}(\tau-\Delta) \int \sigma\left(f_{D R} t_{o}\right) \exp \left(-j 2 \pi f_{D R} \Delta t_{o}\right) d t_{o} \\
& =s_{p}(\tau-\Delta) S_{\sigma^{o}}\left(f_{D R} \Delta\right)
\end{aligned}
$$

where $S_{\sigma^{\circ}}$ is the Fourier spectrum of $\sigma^{\circ}$. Eq. 8 applies for a known $\sigma^{\circ}(t)$. However, $\sigma^{\circ}(t)$ itself is a stochastic process, and the spectral coefficient in $\mathrm{Eq} .8$ has zero average, so no useful information is obtained by averaging $s_{c}(\tau)$. However, when $s_{c}(\tau)$ is detected before it is averaged over the range ensemble, the variance is obtained

$E\left\{\mid s_{c}(\tau)^{2}\right\}=\iiint \int E\left\{\sigma_{c}^{*}\left(t_{0}\right) \sigma_{d}\left(t_{1}\right) \sigma_{c}\left(t_{2}\right) \sigma_{c}^{*}\left(t_{3}\right)\right\}$.

$\exp \left(j 2 \pi\left(f_{D d}\left(t_{0}-t_{1}-t_{2}+t_{3}\right)-\frac{1}{2} f_{D R} \Delta\left(t_{0}+t_{1}-t_{2}-t_{3}\right)\right)\right)$.

$\mathrm{s}_{\mathrm{p}}\left(\tau-\Delta+\mathrm{t}_{0}-\mathrm{t}_{1}\right) \mathrm{s}_{\mathrm{p}}^{*}\left(\tau-\Delta+\mathrm{t}_{2}-\mathrm{t}_{3}\right) d \mathrm{dt}_{\mathrm{o}} \mathrm{dt}_{1} \mathrm{dt}_{2} \mathrm{dt}_{3}$

Now, by using the basic property of samples, $z_{i}$, from zero mean complex Gaussian processes [5]

$E\left\{z_{0}^{*} z_{1} z_{2} z_{3}^{*}\right\}=E\left\{z_{0}^{*} z_{2}\right\} E\left\{z_{1} z_{3}^{*}\right\}+E\left\{z_{0}^{*} z_{1}\right\} E\left\{z_{2} z_{3}^{*}\right\}$

and subsequently applying Eq. 1, the ensemble average of the power detected correlation function is found to be

$E\left\{\left|s_{c}(\tau)\right|^{2}\right\}=\iint \sigma^{o}\left(t_{0}\right) \sigma^{o}\left(t_{1}\right)\left|s_{p}\left(\tau-\Delta+t_{o}-t_{1}\right)\right|^{2} d t_{o} d t_{1}+$
$\iint \sigma^{\circ}\left(t_{0}\right) \sigma^{\circ}\left(t_{2}\right) s_{p}(\tau-\Delta)^{2} \exp \left(-j 2 \pi f_{D R} \Delta\left(t_{o}-t_{2}\right)\right) d t_{o} d t_{2}$

This is for a known $\sigma^{\circ}(t)$. When taking the ensemble average in range and simplifying, the result becomes (* means convolution)

$\mathrm{E}\left(\mid \mathrm{sc}_{\mathrm{c}}(\tau)^{2}\right\}=\mid \mathrm{s}_{\mathrm{p}}(\tau-\Delta)^{2} *\left(\mathrm{R}_{\sigma}(\tau)+\delta(\tau) \mid \mathrm{s}_{\sigma}\left(\mathrm{f}_{\mathrm{DR}} \Delta\right)^{2}\right)$

The spectrum is

$\left.\mathcal{F}\left\{\mathrm{E} \mid \mathrm{s}_{\mathrm{c}}(\tau)^{2}\right\}\right)=\mathcal{F}\left\{\mathrm{s}_{\mathrm{p}}(\tau-\Delta)^{2}\right\rangle\left(\left|\mathrm{S}_{\sigma}(\mathrm{f})^{2}+\right| \mathrm{s}_{\sigma}\left(\mathrm{f}_{\mathrm{DR}} \Delta\right)^{2}\right)$

With the optimum frequency shift, $\mathrm{f}_{\mathrm{DR}} \Delta$ is about half the pulse repetition frequency, and since for most scenes $S_{\sigma^{\circ}}$ is narrow compared to the spectrum of $\left.l s_{p}(t)\right|^{2}$, the last terms in Eqs. 12 and 13 are the smaller ones. This means that the theoretical perfor- 


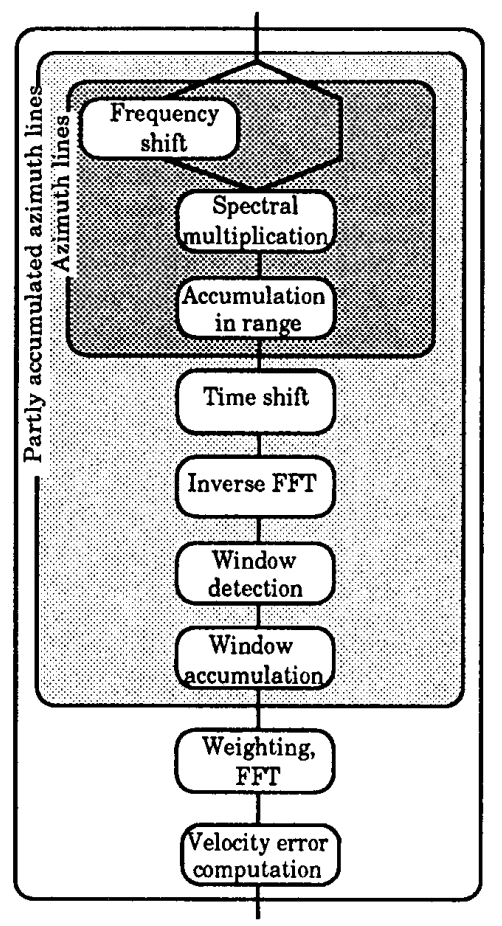

Fig. 2. Shift-and-correlate implementation.

mance of the SAC algorithm is very similar to that of the map drift algorithm. The ensemble average of the map drift correlation function differs at two points as it can be shown by using the same approach as applied in this section. Firstly the smaller terms in Eqs. 12 and 13 are missing, and secondly $s_{p}$ is replaced by a function involving the entire SAR processor impulse response, not only the encoding of the raw data. The latter issue implies that the correlation function broadens if the processor does not focus the SAR image properly.

\section{SAC IMPLEMENTATION}

Fig. 1 shows the implementation of the SAC algorithm. The input is the Doppler spectrum (computed as part of an ordinary rangeDoppler SAR processing). In order to perform the frequency shift and cross-correlation one half of the spectrum is simply shifted relative to the other and multiplied by the complex conjugate of that.

The averaging in the range direction is implemented in two stages. The first stage accumulates the complex correlation functions over small range intervals, e.g. 32 cells, while the second stage detects and averages these partly accumulated correlation functions. In this way the amount of computations is greatly reduced, while the whole range ensemble is still taken into account and enough complex correlation functions are computed to provide a good estimate of their variance, $\left|s_{c}(t)\right|^{2}$.

Since the Doppler rate, and hence the peak position changes with range, a smearing results if the averaging extends over too many range cells. Based on an a priory estimate of the along track velocity, $v_{p}$, the partly accumulated correlation functions are time shifted to an approximate zero peak offset. This time shift is implemented in the frequency domain [3], while the detection and the subsequent averaging are applied in the time domain, and only to a window around the correlation peak.

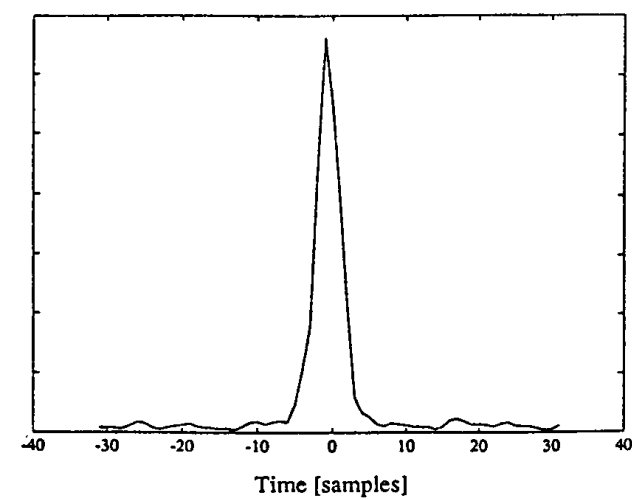

Fig. 3. Correlation peak.

The resulting correlation function is weighted, and its peak position is found from the slope of the phase spectrum. This slope is finally used to compute the difference between $v_{p}$ and the SAC velocity estimate.

It is noted that only one spectral multiplication (cross-correlation) and one complex accumulation, which are both very simple operations, are applied to each and every azimuth line. The computationally expensive FFT operations are applied less frequently. For an estimation window of 2048 samples in azimuth the SAC algorithm calls for a total of about 12000 real operations per azimuth line [3]. Assuming that only two different processing velocities are applied in the map drift algorithm the corresponding figure for that is about 700000 , i.e. over a factor of 50 more.

\section{PERFORMANCE EVALUATION}

Tests have been conducted on data acquired with the Danish airborne SAR [6] including both urban and agricultural scenes. Fig. 3 shows a correlation peak (after range accumulation) typical of the agricultural scene, and the corresponding power spectrum and phase spectrum are shown in Fig. 4 and 5, respectively. With both the SAC algorithm and the map drift algorithm velocity estimates have been obtained from adjacent sub-scenes covering the entire scenes. The sub-scene size is 2048 pixels in azimuth and 512 in range. Table 1 compares the standard deviation of these estimates with the velocity accuracy $\rho_{v}$ needed to achieve an azimuth resolution of $\rho_{\mathrm{a}}$ with a quadratic phase error less than $\pi / 4$

$\rho_{v}= \pm \frac{v \rho_{a}^{2}}{\lambda R}$

For both algorithms the size of the correlation peak is taken as a quality parameter and used to weight the velocity estimates. Table 1 shows that the accuracy of the SAC and map drift algorithms are about the same, and in both cases it is better than required by Eq. $14\left(\rho_{\mathrm{a}}=2 \mathrm{~m}\right)$.

\begin{tabular}{|l|c|c|}
\hline Scene & Urban & Agricultural \\
\hline SAC std. & $0.47 \mathrm{~m} / \mathrm{s}$ & $0.63 \mathrm{~m} / \mathrm{s}$ \\
\hline Map drift std. & $0.37 \mathrm{~m} / \mathrm{s}$ & $0.73 \mathrm{~m} / \mathrm{s}$ \\
\hline Q.cf. Eg. 1 & $0.68 \mathrm{~m} / \mathrm{s}$ & $0.80 \mathrm{~m} / \mathrm{s}$ \\
\hline
\end{tabular}

Table 1. Accuracy of autofocus algorithms. 


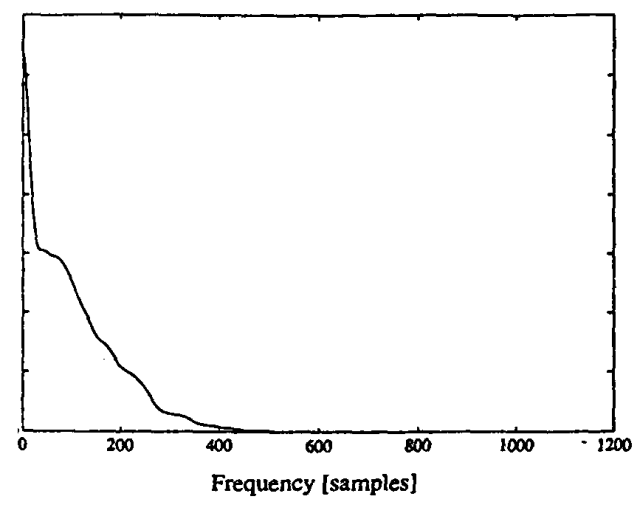

Fig. 4. Power spectrum.

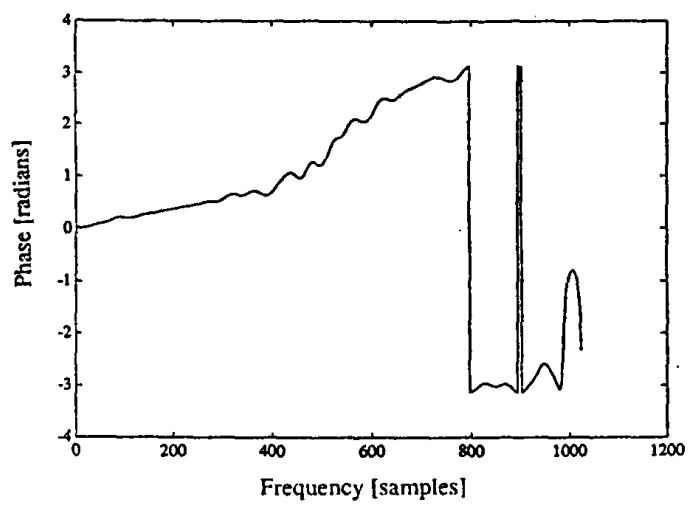

Fig. 5 Phase spectrum.

\section{CONCLUSIONS}

A new autofocus algorithm with a very low computational complexity has been presented. Although based on a fundamentally different principle, this "shift-and-correlate" (SAC) algorithm has some similarity with the traditional map drift algorithm. Theoretically the two algorithms have comparable accuracies, and tests confirm that this is also the case in practice. Both algorithms are able to meet the requirement to a maximum quadratic phase error of $\pi / 4$. The great advantage of the SAC algorithm is that it calls for about 50 times fewer arithmetic operations. It is a single-pass algorithm unlike the map drift and the class of contrast optimization algorithms, which are all iterative. For these reasons it is practicable to apply the SAC algorithm to the entire imagery, not only to sub-windows, and in general the algorithm is very suitable for real-time processing. Thus the Danish high resolution real-time processor [7], which is expected to be operational in the middle of 1992, will adopt the SAC algorithm

\section{ACKNOWLEDGEMENT}

The author would like to acknowledge Dr. Søren Nørvang Madsen, Jet Propulsion Laboratory, who is the originator of the more stringent statistical description presented in Section 3. Also, his encouragement is appreciated.

\section{REFERENCES}

[1] I.P. Finley, and J.W. Wood, "An Investigation of SAR Autofocus", Memorandum 3790, Royal Signals and Radar Establishment, England, 1985.

[2] J.C. Curlander, C. Wu, A. Pang, "Automated Preprocessing of Spaceborne SAR Data", Proceedings of IGARSS'82, pp. FA-1/3.11-6, Munich, June 1982.

[3] J. Dall, "A New Frequency Domain Autofocus Algorithm for SAR", Proceedings of the International Geoscience and - 1072, Helsinki, June, 1991.

[4] S.N. Madsen, "Spectral Properties of Homogeneous and Nonhomogeneous Radar Images", IEEE Transactions on Aerospace and Electronic systems, Vol. AES-23, No. 4, pp. 583-588, July, 1987.

[5] I.S.Reed, "On a moment theorem for complex Gaussian processes", IRE Transactions on Information Theory, pp. 194-195, April, 1962.

[6] S.N. Madsen, E.L. Christensen, N. Skou, J. Dall, "The Danish SAR System; Design and Initial Test", IEEE Transactions on Geoscience and Remote Sensing, Vol. 29, No. 3, pp. 417 - 426, May, 1991.

[7] J. Dall, J.H. Jørgensen, E.L. Christensen, S.N. Madsen, "The Real-Time processor for the Danish Airbome SAR", Accepted for publication in the IEE Proceedings part $F$. 